\title{
SUSCEPTIBILIDAD A Fusarium verticillioides (SACC.) NIRENBERG EN LA POBLACIÓN DE MAÍZ MPB-FCA 856
}

\author{
Daniel Antonio Peiretti-Uzal2, María Cristina Nazar-Lovera ${ }^{2}$, Carlos Alberto Biasutti-Valenzano², \\ Laura María Giorda-Lerda ${ }^{3}$
}

\begin{abstract}
RESUMEN
Susceptibilidad a Fusarium verticillioides (Sacc.) Nirenberg en la población de maíz MPB-FCA 856. Se caracterizó la variabilidad genética de caracteres de la mazorca y susceptibilidad al desarrollo de la podredumbre de la mazorca (Fusarium verticillioides (Sacc.) Nirenberg) en la población de maíz MPB-FCA 856. En Córdoba, Argentina, durante la estación primavera verano 2004/2005, 120 medio hermanos, un híbrido resistente (HR) y otro susceptible (HS), fueron evaluados en un ensayo en bloques completos aleatorizados, con tres repeticiones. Las parcelas fueron dos surcos de $5 \mathrm{~m}$ de longitud a 57.000 plantas/ha. En floración, los estigmas de cinco plantas elegidas al azar en cada tratamiento, fueron inoculadas en dos oportunidades con el aislamiento P364 en solución conidial $\left(1 \times 10^{6} \mathrm{ml}^{-1}\right)$. En madurez fisiológica se midieron seis caracteres de la mazorca. El daño de 96 progenies fue atribuido a $F$. verticillioides. Se calcularon las varianzas y covarianzas y se estableció la interrelación entre todas las variables. Entre los testigos, HR fue significativamente menos dañado que el $76 \%$ de las familias. Los valores de heredabilidad fueron bajos, principalmente para el grado de susceptibilidad $\left(\mathrm{h}^{2}=0,0675\right)$. Los coeficientes de variación genética sugieren la selección por resistencia $(14,98 \%)$ y por la longitud de las brácteas $(26,13 \%)$. Una mayor longitud de las brácteas y el mayor volumen de los granos, disminuyó significativamente $(\mathrm{P}<0,01)$ el grado de susceptibilidad.
\end{abstract}

Palabras claves: Maíz, Fusarium, podredumbre, heredabilidad, correlación.

\begin{abstract}
Susceptibility to Fusarium verticillioides (Sacc.) Nirenberg in the MPB-FCA 856 corn population. The genetic variability of corn ear traits and the sensitivity to corn ear (Fusarium verticillioides (Sacc.) Nirenberg) putrefaction were characterized in the "MPB-FCA 856" corn. The susceptibility-resistance of seeds of 120 half-sibling families, a resistant hybrid $(\mathrm{RH})$ and a sensitive one $(\mathrm{SH})$ was evaluated in a randomized complete sections trial with three repetitions, in Córdoba, Argentina, during spring and summer seasons of 2004/2005. Plots were two furrows of 5 $\mathrm{m}$ in length with 57,000 plants/ha. In the flowering period, stigmata of five randomly chosen plants in each treatment were inoculated twice with P364 insulation in conidial solution $\left(1 \times 10^{6} \mathrm{ml}^{-1}\right)$. Regarding physiological maturity, six corn ear traits were measured. The damage produced to 96 progenies was attributed to $F$. verticillioides. Variances and covariances were calculated and the inter-relationship between all variables was established. Among controls, RH was significantly less damaged than the $76 \%$ of the families. Heritability values were low mainly for sensitivity degree $\left(h^{2}=0.0675\right)$. Genetic variation coefficients encouraged selection by resistance $(14.98 \%)$ and by the increase in bract length $(26.13 \%)$. From the inter-relationship analysis it could be deduced that, increasing bract length and grain volume, sensitivity degree decreases significantly $(\mathrm{P}<0.01)$.
\end{abstract}

Key words: Corn, Fusarium, putrefaction, heritability, correlations.

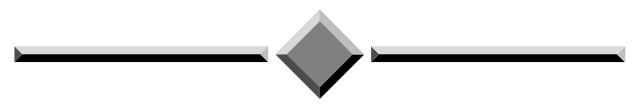

\footnotetext{
1 Recibido: 30 de noviembre, 2006. Aceptado: 29 de agosto, 2007. Programa Interinstitucional PICTOR: No 2002-00012. Disminución de las pérdidas en la producción agroindustrial por la presencia de micotoxinas. Director Héctor Ramón Rubenstein (República Argentina) - Proyecto E: Maíz. Facultad de Ciencias Agropecuarias de la Universidad Nacional de Córdoba.

2 Cátedra de Mejoramiento Genético Vegetal. Facultad de Ciencias Agropecuarias de la Universidad Nacional de Córdoba, Argentina. Correos electrónicos: dapeiret@agro.uncor.edu, marnazar@agro.uncor.edu, cbiasutti@agro.uncor.edu, respectivamente.

3 Cátedra de Fitopatología. Facultad de Ciencias Agropecuarias de la Universidad Nacional de Córdoba. Argentina. Correo electrónico: lgiordam@agro.uncor.edu
} 


\section{INTRODUCCIÓN}

El principal agente patógeno causante de la podredumbre de la mazorca de maíz (Zea mays L.) es Fusarium verticillioides (Sacc.) Nirenberg, se inicia con la formación de micelios blancos, que van descendiendo desde la punta de la mazorca y dan una coloración rojiza a rosada a los granos infectados. Seguidamente se producen micotoxinas, particularmente las fumonisinas que tienen efectos tóxicos cuando son consumidos por humanos y animales (Levin et al. 2003; Bush, et al. 2004). Fusarium verticillioides ataca en todos los estados de crecimiento de la planta de maíz y a diferentes partes de la misma induciendo enfermedades de pre y postcosecha que causan reducción de rendimientos y afectan la calidad de la semilla (Schulthess et al. 2002). Algunas cepas de Fusarium verticillioides producen las infecciones asintomáticas de la semilla, la cual se transmite a la plántula afectando su emergencia (Yates et al. 1997). En las infecciones asintomáticas las hifas colonizan los espacios intercelulares, a diferencia de las infecciones sintomáticas que se encuentran tanto en los espacios Inter como intracelulares (Yates y Jaworshi 2000; Oren et al. 2003). La infección asintomática en plantas dificulta la selección por resistencia, basada en evaluaciones visuales (Giorda y Peiretti 2006; Duvick 2001).

La susceptibilidad diferencial de los genotipos de maíz al ataque de este hongo, se conoce a nivel de cultivares inscriptos (Presello et al. 2005), sin embargo es necesario establecer la variabilidad que existe dentro de cada población cuando ésta debe ser sometida al proceso de selección. Asimismo, resulta necesario establecer las posibles interrelaciones entre los diferentes grados de susceptibilidad y la variación de otros caracteres implicados en la cobertura de la mazorca. En particular, la cantidad de hojas que envuelven la mazorca y la prolongación de las brácteas en más de 5 $\mathrm{cm}$ de la punta de la mazorca, que han sido reportados como asociados con la prevención de la resistencia a humedad y el desarrollo de la pudrición de la mazorca (Mejía Contrera et al. 1983).

El objetivo de este trabajo fue cuantificar y caracterizar la variabilidad genética de caracteres de la mazorca y su relación con la susceptibilidad al desarrollo de la podredumbre de la mazorca ocasionada por Fusarium verticillioides (Sacc.) Nirenberg, en una población de maíz utilizada como material base de selección en proyectos de mejora genética.

\section{MATERIALES Y MÉTODOS}

El material original fue la población de polinización libre de maíz colorado duro MPB-FCA 856 (Peiretti et al. 1988), de la cual se obtuvieron al azar 120 familias de medio hermanos visiblemente sanas. Se incluyeron dos híbridos reportados por Presello, et al. (2005), uno como resistente (HR) y otro como susceptible (HS). Las semillas de cada mazorca, se desinfectaron con una solución de hipoclorito de sodio al 5\%, se lavaron con agua esterilizada y secaron con una corriente de aire frío hasta un $10 \%$ de humedad. Las muestras así acondicionadas fueron almacenadas a $5^{\circ} \mathrm{C}$. En octubre de 2004, en el Campo Escuela de la Facultad de Ciencias Agropecuarias de la Universidad Nacional de Córdoba, distante $20 \mathrm{~km}$ de la ciudad de Córdoba ( $64^{\circ}$ longitud oeste, $31,5^{\circ}$ latitud sur, y 425 msnm), como única localidad, se implantó un ensayo de evaluación de la susceptibilidad-resistencia.

Las características agroclimáticas de esta localidad, corresponden a las de una zona templada con una duración de 14 horas 10 minutos para el día más largo que ocurre en el solsticio de verano ( 22 de diciembre). Mientras que en el solsticio de invierno (22 de junio) ocurre el día más corto del año con una duración de 9 horas 50 minutos. La amplitud térmica del aire en los meses que correspondieron al ensayo, estuvo dada por $17,6{ }^{\circ} \mathrm{C}$ en promedio para el mes de octubre y por $22,69{ }^{\circ} \mathrm{C}$ en promedio para el mes de febrero. La temperatura promedio del ciclo fue de $21,64{ }^{\circ} \mathrm{C}$. Las precipitaciones acumularon, en el ciclo de ensayo, una cantidad de $531 \mathrm{~mm}$, sobre el monto anual característico de la zona de $772 \mathrm{~mm}$. La radiación media fue de $20,82 \mathrm{MJ} / \mathrm{m}^{2}$. El suelo corresponde al tipo haplustol éntico.

El arreglo de las parcelas correspondió a un diseño en bloques completos aleatorizados, con tres repeticiones. Las parcelas experimentales estuvieron constituidas por dos surcos de $5 \mathrm{~m}$ de longitud, separados a 0,7 . En cada surco se sembraron 50 semillas, y se ralearon al momento de desarrollarse la tercer hoja a 20 plantas por surco tratando de mantener la uniformidad de espaciamiento. La unidad experimental 
fueron cinco plantas de cada tratamiento, elegidas al azar dentro de la parcela experimental. En el momento de la floración, cuando los estigmas asomaron unos $5 \mathrm{~cm}$ por encima de las brácteas de la inflorescencia fueron inoculados con $4 \mathrm{ml}$ de una solución conidial a una concentración de 1 x $10^{6}$ conidios $/ \mathrm{ml}$. La fuente de inóculo fue el aislamiento P364, y fue indicada como productora de fumonisinas in vivo e in vitro con un nivel de agresividad intermedio. La inoculación se realizó en dos oportunidades durante el mes de marzo.

En el momento de la madurez fisiológica cuando la humedad del grano alcanzó el 20\%, se obtuvieron los siguientes caracteres de la mazorca:

a. Longitud en que las brácteas sobrepasaron a la mazorca en cm (LT).

b. Cantidad de hojas que envuelven a la mazorca (CH).

c. Longitud de la mazorca sin brácteas en $\mathrm{cm}$ (LM).

d. Volumen de grano, promedio obtenido sobre una muestra de 100 medido en $\mathrm{ml}$ (VG).

e. Porcentaje de podredumbre de la mazorca (GS). Se consideró la relación entre granos afectados y la totalidad de los granos de la mazorca como un estimador del grano de susceptibilidad.

A las muestras de los granos afectados se les identificó el agente causal. El daño de 96 familias fue atribuido a $F$. verticillioides. En los análisis estadísticos, los tratamientos cuya podredumbre se debió a otras causas fueron considerados no dañados. Para el análisis de los datos se utilizó el Programa Estadístico Infostac (2006). Se calcularon varianzas y covarianzas, solo para el porcentaje de podredumbre de la mazorca (GS). Mientras que, para establecer las interrelaciones entre todos los caracteres medidos, se incluyeron a todas las variables medidas en un análisis de coeficientes de correlaciones fenotípicas, siguiendo el modelo Pearson. Para la partición de los coeficientes de correlación en efectos directos e indirectos, se utilizó el procedimiento dado por Wright (1934). El análisis de los componentes de la variabilidad fenotípica observada en esta muestra de genotipos, se realizó siguiendo el análisis clásico dado por Mather y Jink (1971). En donde la correspondencia en las expectaciones de los productos medios del análisis de covarianzas de los cuadrados medios del análisis de la varianza permiten establecer estimaciones genéticas. El modelo correspondió al siguiente criterio presentado en el Cuadro 1.
Cuadro 1. Modelo de análisis de variancias y covariancias. Córdoba, Argentina. 2004.

\begin{tabular}{|c|c|c|c|}
\hline FV & GL CM & & ECM \\
\hline Repeticiones (R) & 2 & & \\
\hline Familias (F) & 119 & M1 & $\begin{array}{l}\hat{O}^{2}{ }_{\mathrm{E}}+\mathrm{r} \hat{o}^{2}{ }_{\mathrm{w}}+\mathrm{n} \hat{o}^{2}{ }_{\mathrm{e}}+ \\
\mathrm{nr} \hat{o}^{2}{ }_{\mathrm{F}}\end{array}$ \\
\hline $\mathrm{R} \times \mathrm{F}$ & 238 & M2 & $\hat{o}_{\mathrm{E}}^{2}+\mathrm{r} \hat{o}^{2}{ }_{\mathrm{w}}+\mathrm{n} \hat{o}_{\mathrm{e}}^{2}$ \\
\hline $\mathrm{N} \times \mathrm{F}$ & 476 & M3 & $o^{2}{ }_{E}+r o^{2}{ }_{W}$ \\
\hline Error & 964 & M4 & $\mathrm{o}^{2} \mathrm{E}$ \\
\hline Total & 1.799 & & \\
\hline
\end{tabular}

Donde:

R x F: Interacción repeticiones por familias (error entre parcelas)

$\mathrm{N} x$ F: Plantas dentro de familias

$\mathrm{O}^{2} \mathrm{E}$ : Varianza residual

$\mathrm{o}^{2} \mathrm{w}$ : Varianza dentro de familias

$o^{2}{ }_{\mathrm{e}}$ : Varianza ambiental del error entre familias

$\mathrm{o}^{2} \mathrm{~F}$ : Varianza entre familias de medio hermanos

r: Repeticiones

$\mathrm{n}$ : Cantidad de plantas por familias.

Siguiendo el modelo, la varianza aditiva se estimó multiplicando por cuatro al componente de varianza entre familias (ó2F). Mientras que la varianza fenotípica ó2P se estimó por la suma de los componentes entre familias de interacciones familias por repeticiones, de plantas dentro de familias y el error residual.

$$
\text { ó } 2_{\mathrm{P}}=\text { ó } 2_{\mathrm{F}}+\text { ó } 2_{\mathrm{e}}+o ́ 2_{\mathrm{w}}+o ́ 2_{\mathrm{E}}
$$

La heredabilidad fue estimada por el coeficiente de varianza aditiva (ó2 2 ) sobre ó $2 \mathrm{P}$. Además, se calculó el coeficiente de variación genética $(\mathrm{CV}(\mathrm{G}))$ por el siguiente cociente

$$
\mathrm{CV}(\mathrm{G})=\left(o^{2} \mathrm{P}-\text { ó } 2_{\mathrm{E}}\right) / \mathrm{mg}
$$

en donde mg es la media general del ensayo, excluyendo a los cultivares testigos.

\section{RESULTADOS}

El análisis de la varianza para cada uno de los caracteres analizados dieron valores de $\mathrm{F}$ altamente significativos $(\mathrm{P}<0,01)$ para LT y GS, y significativos $(\mathrm{P}<0,05)$ para LM y VG (Cuadro 2). Con relación a los testigos, solo HR (altamente resistente) se mostró significativamente menos dañado que el $76 \%$ de las familias. 
Cuadro 2. Valores de media general de las familias de familias de medio hermanos, sus heredabilidades y coeficientes de variación para los caracteres analizados en el cultivar MPB-FCA 850. Córdoba, Argentina. 2005.

\begin{tabular}{llcc}
\hline Variable & $\begin{array}{c}\text { Media } \\
\text { general }\end{array}$ & Heredabilidad & $\begin{array}{c}\text { Coeficiente } \\
\text { de variación } \\
\text { genética }(\%)\end{array}$ \\
\hline LT & $17,4^{* *}$ & 3,88 & 26,13 \\
CH & $12,9^{\mathrm{NS}}$ & 9,63 & 9,42 \\
$\mathrm{LM}$ & $14,7^{*}$ & 6,74 & 31,78 \\
$\mathrm{VG}$ & $87,8^{*}$ & 22,61 & 6,51 \\
$\mathrm{GS}$ & $32,4^{* *}$ & 6,87 & 14,98 \\
\hline
\end{tabular}

* Diferencias significativas $(\mathrm{P}<0,05)$.

** Diferencias altamente significativas $(\mathrm{P}<0,01)$. ns Diferencias no significativas.

LT = Longitud de brácteas; $\mathrm{CH}=$ Cantidad de Hojas; $\mathrm{LM}=$ Longitud de mazorca; VG = Volumen de 100 granos; GS = Relación granos afectados/total de granos de la mazorca.

Los valores de heredabilidad para cada carácter, si bien fueron cuantitativamente bajos, corresponden a valores normales en este tipo de poblaciones. En general, se indica con ello que la variación generada por el ambiente es muy importante, particularmente en el caso del grado de susceptibilidad de los genotipos. Se debe tomar en cuenta que en el análisis se consideraron todas las familias, aún aquellas cuyos daños fueron atribuidos a otras causas y a las cuales se les supuso resistente. Esto debió introducir un error en la partición de los componentes, que puede ser reparado en mediante diseños que permitan considerarlos. Por su parte los coeficientes de variación genética por si mismos dan valores alentadores para selección por resistencia y también para aumentar la longitud de las brácteas que envuelven a la mazorca.

La relación entre los caracteres, a través de la matriz de coeficientes de correlación, se muestra en el Cuadro 3. Las asociaciones que se dieron entre los caracteres de la mazorca corresponden a las características varietales de MPB-FCA 856, ya discutidos en otras experiencias (Peiretti et al. 1988). Los valores y significancias, se muestran con el solo fin de indicar la relación entre los caracteres medidos en la mazorca y el grado de susceptibilidad a la podredumbre causada por
Cuadro 3. Coeficientes de correlaciones fenotípicas entre los caracteres analizados en la población de maíz MPBFCA 856. Córdoba, Argentina. 2005.

\begin{tabular}{lccccc}
\hline & LT & CH & LM & VG & GS \\
\hline LT & 1 & $-0,068^{\text {ns }}$ & $-0,703^{* *}$ & $-0,163^{* *}$ & $-0,451^{* *}$ \\
CH & & 1 & $0,415^{* *}$ & $0,237^{* *}$ & $0,126^{*}$ \\
LM & & & 1 & $-0,018^{\text {ns }}$ & $0,374^{* *}$ \\
VG & & & & 1 & $-0,228^{* *}$ \\
GS & & & & & 1 \\
\hline
\end{tabular}

* Asociaciones significativas $(\mathrm{P}<0,05)$.

** Asociaciones altamente significativas $(\mathrm{P}<0,01)$.

ns Asociaciones no significativas.

LT = Longitud de brácteas; $\mathrm{CH}=$ Cantidad de Hojas; $\mathrm{LM}=$ Longitud de mazorca; $\mathrm{VG}$ = Volumen de 100 granos; GS = Relación granos afectados/total de granos de la mazorca.

F. vercillioides. Los valores indican que en la medida que aumentó la longitud de las brácteas de la mazorca (LT) y el volumen de los granos (VG), disminuyó el grado de susceptibilidad (GS) en forma altamente significativa. Estos resultados corroboran la mención que se hace en la publicación de Mejía Contrera (1983). En el mismo sentido, coeficiente de correlación positiva y altamente significativa que mostró la longitud de mazorca (LM) con GS, está explicado por la correlación inversa entre LM y LT.

En el Cuadro 4, se presentan los efectos directos e indirectos en que se han particionado los coeficientes de correlación de los caracteres medidos con respecto al grado de susceptibilidad de las familias a la podredumbre de la mazorca. Los coeficientes de correlación de los caracteres longitud de las brácteas de la mazorca (LT), cantidad de hojas $(\mathrm{CH})$ y volumen del grano sobre la susceptibilidad (GS), en forma prioritaria, se originan en sus respectivos efectos directos, considerando poco significantes a sus efectos indirectos a través de los otros caracteres. En cuanto al carácter longitud de la mazorca (LM), el efecto principal que facilita el incremento de la susceptibilidad, está dado en forma indirecta a través de longitud de las brácteas (LT). La explicación lógica es que, cuando la longitud de la mazorca aumenta, existe mayor posibilidad de que su extremo distal quede descubierto facilitando el desarrollo del patógeno. 
Cuadro 4. Partición de los coeficientes de correlación (r) en efectos directos e indirectos sobre porcentaje de podredumbre de $F$. verticillioides en maíz. Córdoba, Argentina. 2005.

\begin{tabular}{|c|c|c|}
\hline \multicolumn{3}{|c|}{ Efectos de LT sobre GS } \\
\hline & Directo & $-0,62$ \\
\hline & Indirecto a través de $\mathrm{CH}$ & $-0,02$ \\
\hline & Indirecto a través de LM & 0,12 \\
\hline & Indirecto a través de VG & 0,06 \\
\hline & $\mathrm{r}$ total & $-0,45$ \\
\hline \multicolumn{3}{|c|}{ Efectos de CH sobre GS } \\
\hline & Directo & 0,25 \\
\hline & Indirecto a través de LT & 0,04 \\
\hline & Indirecto a través de LM & $-0,07$ \\
\hline & Indirecto a través de VG & $-0,09$ \\
\hline & $\mathrm{r}$ total & 0,13 \\
\hline \multicolumn{3}{|c|}{ Efectos de LM sobre GS } \\
\hline & Directo & $-0,17$ \\
\hline & Indirecto a través de LT & 0,43 \\
\hline & Indirecto a través de $\mathrm{CH}$ & 0,10 \\
\hline & Indirecto a través de $\mathrm{VG}$ & 0,01 \\
\hline & $\mathrm{r}$ total & 0,37 \\
\hline \multicolumn{3}{|c|}{ Efectos de VG sobre GS } \\
\hline & Directo & $-0,39$ \\
\hline & Indirecto a través de LT & 0,10 \\
\hline & Indirecto a través de $\mathrm{CH}$ & 0,06 \\
\hline & Indirecto a través de VG & $3,1 \mathrm{E}-03$ \\
\hline & $\mathrm{r}$ total & $-0,23$ \\
\hline
\end{tabular}

LT = Longitud de brácteas; $\mathrm{CH}=$ Cantidad de Hojas; $\mathrm{LM}=$ Longitud de mazorca; $\mathrm{VG}=$ Volumen de 100 granos; $\mathrm{GS}$ = Relación granos afectados/total de granos de la mazorca.

\section{CONCLUSIONES}

Existió variabilidad genética en la población MPBFCA 856, estimada a través de los coeficientes de variación genética, que indica la posibilidad de seleccionar genotipos que muestren resistencia al desarrollo de la podredumbre de la mazorca ocasionada por $F$. verticillioides.

Los métodos de mejora poblacional deberán estar orientados a seleccionar entre y dentro de familias, con base en pruebas de progenies. Esto en razón de que los valores estimados de heredabilidad, revelan que la variación en el ambiente provoca una fuerte modificación de la expresión de los genotipos.
La selección puede hacerse a través de caracteres componentes de la mazorca, principalmente la relación que involucra a la longitud de la mazorca y de las brácteas que la envuelven. La longitud de las brácteas, por encima del extremo distal de la espiga, ha mostrado tener una correlación negativa con la susceptibilidad al desarrollo de la enfermedad.

\section{LITERATURA CITADA}

BUSH, B. J.; CARSON, M. L.; CUBETA, M. A.; HAGLER, W.M.; PAYN, G.A. 2004. Infection and fumonisin production by Fusarium verticillioides in developing maize kernels. Phytopathology 94:99-93.

DUVICK, J. 2001. Prospects for reducing fumonisin contamination of maize trough genetic modification. Environmental health perspectives 109(2):337-342.

GIORDA, L.; PEIRETTI, D. 2006. Respuesta de germoplasma de maíz a infecciones por Fusarium verticillioides y contaminación con fumonisinas. In: Rubinstein, H.R. ed. Micotoxinas: Impacto en la producción y salud humana y animal. Cap. 5. Universidad Nacional de Córdoba. p. 125-138.

INFOSTAT. 2005. Grupo Infostat/FCA. Universidad Nacional de Córdoba. Ed. Brujas, Córdoba, Argentina.

LEVIN, L.; RIDAO, A.; CASTAÑO, F. 2003. Fusariosis de la espiga en el maíz. INTA: $20^{\text {a }}$ Jornada de actualización profesional en cultivos de verano. Mar del Plata, 19 de setiembre de 2003. p. 165.

MATHER, K.; JINK, J.L. 1971. Biometrical genetics. Chapman and Hall. p. 379.

MEJIA, A.; MARQUEZ, F.; CARBALLO, A. 1983. Cobertura de la mazorca de maíz: Heredabilidad y correlación con otros caracteres. Agrociencia 54:111-123.

OREN, L.; EZRATI, S.; COHEN, D.; SHARON, A. 2003. Early events in the Fusarium verticillioides-maize interaction characterized by using green fluorescent protein-expressing transgenic isolate. Appl. Envirom. Microbiol. 69(3):695-701.

PEIRETTI, D. A.; BARRANDEGUY, M. A.; BIASUTTI, C. A. 1988. MPB FCA 856, variedad experimental 
de maíz para la zona centro norte de la provincia de Córdoba. IV Congreso Nacional de Maíz. Pergamino (Argentina) 1:64-70.

PRESELLO, D.; BOTTA, G.; IGLESIAS, J.; EYHERABIDE, G. 2005. Efecto de la severidad de síntomas de podredumbre de espiga causada por Fusarium verticillioides sobre el rendimiento y la conservación de fumonisinas en grano. In INTA - MAÍZ: Resultado de experiencias 2004-2005. p. 99-102.

SCHULTHESS, K. F.; CARDWELL, K. F.; GOUNOU, S. 2002. The effect to endophytic Fusarium verticillioides on infestation of two maize varieties by lipidopterous stemborers and coleopteran grain feeders. Phitopathology Vol 92, No 2: 121-128.

WRIGHT, S. 1934. The method of path coefficient. Ann. Math. Stat. 5:161-215.

YATES, I. E.; BACON, C. W.; HINTON, D. M. 1997. Effects of endophytic infection by Fusarium verticillioides on corn growth and cellular morphology. Plant Disease Vol. 81 N $^{\circ}$. 7:723-728.

YATES, I. E.; JAWORSHI, A. J. 200. Differential growth of Fusarium verticillioides relative to tissues from "Silver Queen”, a sweet maize. Can. J. Bot. 78(4):472-480. 